\title{
Side Effect
}

National Cancer Institute

\section{Source}

National Cancer Institute. Side Effect. NCI Thesaurus. Code C2861.

The term was used in various ways in the past, to describe unintended effects of medical intervention, usually negative (unfavourable), but also positive (favourable) ones. It is recommended that this term no longer be used and particularly should not be regarded as synonymous with adverse event or adverse reaction. 